\title{
Hinode/EIS plasma diagnostics in the flaring solar chromosphere
}

\author{
D. R. Graham, L. Fletcher, and I. G. Hannah
}

School of Physics and Astronomy, University of Glasgow, Glasgow G12 8QQ, UK

e-mail: d.graham@astro.gla.ac.uk

Received 16 July 2010 / Accepted 1 June 2011

\begin{abstract}
Context. The impulsive phase of solar flares is a time of rapid energy deposition and heating in the lower solar atmosphere, leading to changes in the temperature, density, ionisation and velocity structure of this region.

Aims. We aim to study the lower atmosphere during the impulsive phase of a flare using imaging and spectroscopic data from Hinode/EIS, RHESSI and TRACE. We place these observations in context by using a wide range of temperature observations from each instrument.

Methods. We analyse sparse raster data from the Hinode/EIS spectrometer to derive the density and line-of-sight velocity in flare footpoints, in a GOES C6.6 flare observed on 05-June-2007. The raster duration was 150s across the centre of a small active region, allowing multiple exposures of the flare ribbons and footpoints. Using RHESSI and Hinode/XRT we test both non-thermal and thermal models for the HXR emission.

Results. During the flare impulsive phase, we find evidence from XRT for flare footpoints at temperatures exceeding 7 MK. We measure the electron number density increasing up to a few $\times 10^{10} \mathrm{~cm}^{-3}$ in the footpoints, at temperatures of $\sim 1.5-2 \mathrm{MK}$, accompanied by small downflows at temperatures below Fe XIII and upflows of up to $\sim 140 \mathrm{~km} \mathrm{~s}^{-1}$ at temperatures above. This is reasonable in the context of HXR diagnostics of the flare electron beam. The electrons inferred have sufficient energy to affect the chromospheric ionisation structure.

Conclusions. EIS sparse raster data coupled with RHESSI imaging and spectroscopy prove useful here in studying the lower atmosphere of solar flares, and in this event suggest heat deposition relatively high in the chromosphere drives chromospheric evaporation while increasing the observed electron densities at footpoints. However, from RHESSI spectral fitting it is not possible to say whether the data are more consistent with a model including a non-thermal beam, or purely thermal model.
\end{abstract}

Key words. Sun: atmosphere - Sun: chromosphere - Sun: flares - Sun: UV radiation - Sun: X-rays, gamma rays

\section{Introduction}

Observationally, the impulsive phase of a solar flare is characterised by a bursty light curve seen in hard X-rays (HXRs), compact footpoints formed in the lower atmosphere, and strong emission in the ultraviolet (UV) and extreme ultraviolet (EUV). This represents the result of the release of stored magnetic energy which is transmitted rapidly to the lower atmosphere. The presence of HXRs is a clear diagnostic of non-thermal electrons in solar flares, which in the "standard flare model" are the agents which transport energy from the corona to the footpoints.

The result of the deposition of this energy in the lower atmosphere is ionisation and plasma heating, and energy loss by plasma expansion, radiation and conduction (though the latter is not generally thought important in the low temperature chromosphere). Heating is clear from the appearance of impulsive flare footpoints in higher energy lines and continuum thermal radiation, at least up to the soft X-ray regime (Mrozek \& Tomczak 2004). In the impulsive phase of a solar flare, ionisation and heating is caused by the non-thermal electrons, which are detectable via their hard X-ray (HXR) radiation, with the non-thermal electrons themselves contributing directly to collisional ionisation and the production of energetic secondary electrons (Hudson 1972; Brown 1973a; Ricchiazzi \& Canfield 1983) as well as collisional heating of ambient electrons (Brown 1973b). In the gradual phase of a flare, footpoint or ribbon heating is thought to be by conductive energy flux from the overlying high-pressure post-flare loops (Antiochos \& Sturrock 1978; An et al. 1983; Czaykowska et al. 2001).

The plasma expansion mentioned above, usually known as chromospheric evaporation, is the re-adjustment of the lower atmospheric plasma towards a new hydrostatic equilibrium following heating. If the atmosphere is unable to radiate away the energy deposited and heats past the peak in the radiative loss curve, it will expand. If in addition the heating timescale is shorter than the hydrodynamic expansion timescale, "explosive" evaporation occurs. According to Fisher et al. (1985b) and other modeling work based on electron beam energy deposition, the transition from gradual to explosive evaporation typically occurs at values around $10^{10} \mathrm{erg} \mathrm{cm}^{-2} \mathrm{~s}^{-1}$. Evaporation has been observed in spatially-resolved observations with the SOHO Coronal Diagnostic Spectrometer (Harrison et al. 1995), and Hinode/EIS (Culhane et al. 2007). It is difficult to make such observations in the flare impulsive phase due to the rarity of having a scanning spectrometer slit on top of the shortlived impulsive phase kernel at the right time. However, Milligan et al. (2006a) did observe strongly blueshifted $\left(\sim 250 \mathrm{~km} \mathrm{~s}^{-1}\right)$ Fe XIX emission (formation temperature $\log T_{\mathrm{e}}=6.9$ ) from footpoints, along with weakly redshifted $\mathrm{He} \mathrm{I}$ and $\mathrm{O} v$ emission, and RHESSI imaging confirmed the location of these flows at the flare HXR footpoints. Much slower "gentle" evaporative flows were also seen (Milligan et al. 2006b). With Hinode/EIS (Culhane et al. 2007), blueshifted emission was observed by Milligan \& Dennis (2009) to be coincident with RHESSI HXR 
footpoints in six emission lines (Fe xIV-XxIV) formed over the temperature range $\log T_{\mathrm{e}}=6.3-7.2$. These upflows were found to scale with temperature reaching speeds of $>250 \mathrm{~km} \mathrm{~s}^{-1}$ in the $\mathrm{Fe}$ xxIv line. A new finding was that material formed at temperatures from $\log T_{\mathrm{e}}=4.7-6.2$ was redshifted by several tens of $\mathrm{km} \mathrm{s}^{-1}$. The origin of these hot downflows is unclear; beam-driven evaporation models predict that impulsive downflows occur below transition region temperatures (e.g. Fisher et al. 1985b; Allred et al. 2005). In addition to high speed flows, Watanabe et al. (2010) have observed electron density enhancements of up to $3 \times 10^{10} \mathrm{~cm}^{-3}$ at evaporation sites.

Chromospheric heating and ionisation during the impulsive phase of a flare is generally interpreted in the context of the collisional thick target model (Brown 1971; Kane \& Anderson 1970) in which non-thermal electrons are continuously accelerated into the chromosphere, where they are stopped collisionally and also radiate HXR bremsstrahlung emission. The spatial distribution of beam heating depends sensitively on the spectral index of the electron distribution, which can be determined well from fitting spectral data from the RHESSI spacecraft (Lin et al. 2002), and also the lower limit to the energy of the nonthermal electrons (the "low energy cutoff"). The value of the latter parameter, if it exists, is notoriously hard to determine from spectral fitting, as it generally occurs within the energy range of a much stronger thermal component. It is nonetheless crucial in beam heating. Should the electron beam penetrate below the fully-ionised layers of the chromosphere then ionisation losses by the beam (which increase the target ionisation fraction), must also be taken into account.

This paper reports of observations during the impulsive phase of a small solar flare using the EIS instrument on Hinode supported by TRACE (Handy et al. 1999), the Ramaty High Energy Solar Spectroscopic Imager (RHESSI Lin et al. 2002), the Hinode Solar Optical Telescope (SOT Tsuneta et al. 2008) and the Hinode X-Ray Telescope (XRT Golub et al. 2007). Of primary interest is the evolution of the electron density at the flare footpoints, and its interpretation in the context of current flare models. The flexibility of Hinode/EIS allows "sparse" rasters which can scan across an active region several times during a flare, with the slit jumping to non-contiguous positions, thus gaining greater spatial coverage and allowing multiple exposures of a single region, but at the expense of spatial information. Studies of this kind have made significant progress in understanding chromospheric evaporation, see Milligan \& Dennis (2009), Watanabe et al. (2010), and Del Zanna et al. (2011). In this paper we present the results of one such sparse raster observation, which has enabled us to study flare ribbons and footpoints at different times in their evolution. After giving an overview of the event and the data in Sect. 2, we present the EIS data in Sect. 3 and the results of the EIS diagnostics in Sect. 4. In Sect. 5 we show the RHESSI analysis, and discuss the physical processes occurring at the footpoints in this event in Sect. 6, ending with conclusions in Sect. 7.

\section{Event overview}

We present here data from a GOES C6.6 class flare from NOAA active region 10960, which took place on June 5th 2007. The GOES 5-min X-Ray data (Fig. 1, upper panel) show the flare beginning at 15:50 UT, peaking at 16:13 UT, and ending at 17:00 UT. The event was observed by EIS on Hinode (Culhane et al. 2007), TRACE (Handy et al. 1999), and RHESSI (Lin et al. 2002). Unfortunately all have missing data across the GOES peak because of either SAA or night-time passage. However,

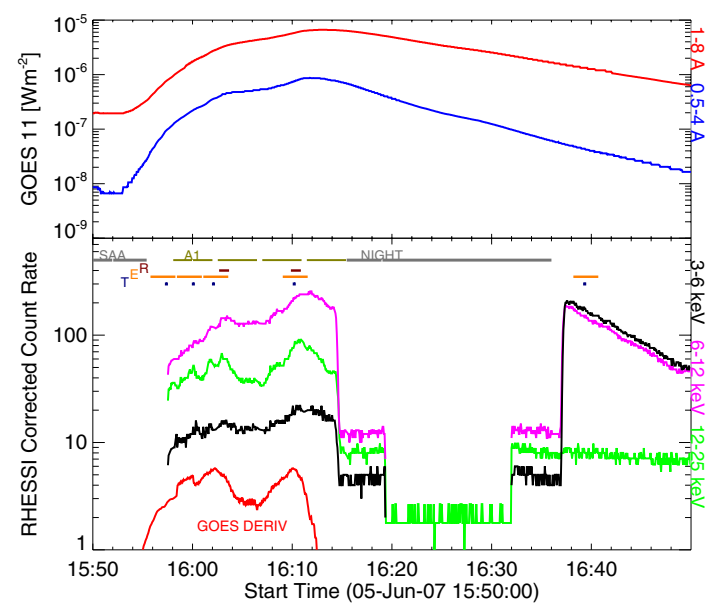

Fig. 1. GOES (top panel) and RHESSI (bottom panel) X-ray light curves of the flare. In the bottom panel the times of the EIS raster, TRACE images and RHESSI images/spectra are shown by the labeled bars. A time derivative of the GOES 1-8 $\AA$ channel is found in red and has been scaled for context purposes. Also shown are the times of RHESSI South Atlantic Anomaly (SAA) passage and Eclipse period, when no data is available, and when the attenuating shutters were deployed (A1).

there is good coverage of some small, early impulsive phase bursts, characterised by two peaks in the RHESSI $12-25 \mathrm{keV}$ light curve, and two coincident peaks in the GOES Soft X-Ray (SXR) derivative (Fig. 1). Steep rises in the SXR flux can often be suggestive of non-thermal electron deposition (Neupert 1968; Dennis \& Zarro 1993), so it is possible that there are two distinct periods of flare heating.

TRACE images in the $171 \AA$ passband show the flare footpoints and ribbon patterns. Figure 2 shows a series of TRACE images cropped to the EIS field of view, with contours corresponding to Hinode/XRT overplotted. The times also correspond to the mid-point of some selected EIS rasters: (1) a frame when the earliest signs of footpoint brightening are seen; (2) as these bright points develop into ribbons; (3) corresponding to the closest EIS raster to the first peak in the RHESSI $12-25 \mathrm{keV}$ band and the GOES X-Ray Derivative; (4) as in the previous frame but for the larger second peak; and (5) a raster in the decay phase straight after the data gap. In the first exposure, the overall morphology of the region is clear. A large patch of moss emission in the centre is bisected by a short filament, and two large fans of field originating in magnetic concentrations can also be seen. The filament does not change substantially during the event and neither do the overlying large TRACE loops, suggesting that this was not an eruptive flare.

In the first TRACE exposure shown, small brightenings become apparent on either side of the filament, spreading along the moss region in the second and third rasters. In the fourth, chromospheric flare ribbons are visible as a distinctive "U" shape in the south region and an inverted " $L$ " shape in the north. Though the TRACE emission is at $171 \AA$, nominally at coronal temperatures, it is clear that in this instance it is from chromospheric heights. This can also be seen by inspecting a Hinode/SOT Ca H band image from the same time (Fig. 3), which shows the upper ribbon (SOT having a smaller fieldof-view). This has the same typical "L" shape. In the last TRACE exposure, after TRACE eclipse, the flare is well into its decay phase and flare loops are visible, which expand as expected. As mentioned before, the filament is apparently not disturbed in its position. 
D. R. Graham et al.: Hinode/EIS flare density diagnostics

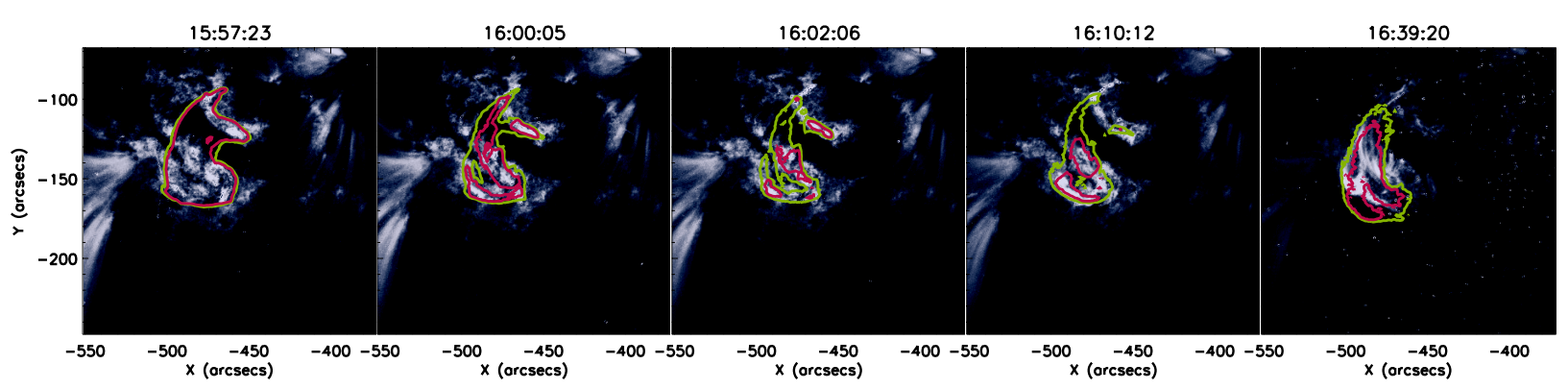

Fig. 2. The flare seen by TRACE at $171 \AA$ over several stages during its evolution. Over plotted are Hinode/XRT contours shown in green and magenta, marking 60\% and 80\% intensity levels. The overall XRT intensity in each image peaks around the time of the 16:10 frame, however the maximum extent of the loop structure shown does not vary greatly.

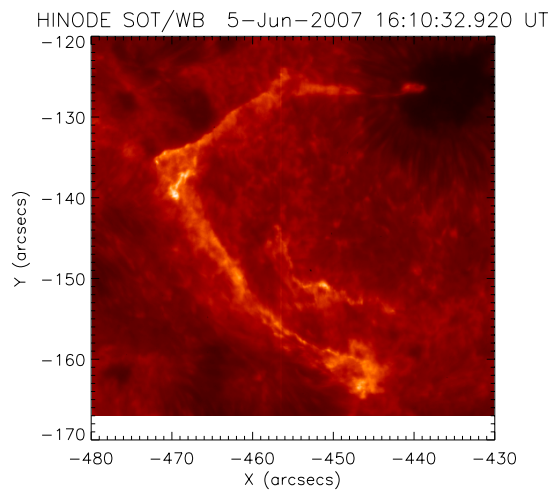

Fig. 3. The northern flare ribbon seen in the Hinode/SOT Ca H line, close to the small peak at 16:11 UT.

High temperature observations of flare loops with Hinode/ XRT Ti-Poly filter (peaking around $7 \mathrm{MK}$ ) highlight plasma in loops above the transition region. The visible edges are defined by the $60 \%$ and $80 \%$ contours of the maximum image intensity in Fig. 2. Two loops are seen growing in the impulsive phase, but at their brightest - the fourth frame - remain bounded by the two bright TRACE $171 \AA$ footpoints in the north, and the single ribbon area in the south. The higher intensity contour surrounds the flare ribbon in the fourth image, suggesting some of this hotter emission is in fact from hot footpoints. Also the observation that most of the XRT loop intensity is contained within the location of TRACE bright points supports our view that TRACE is indeed showing us flare footpoints.

The RHESSI spacecraft also covered the early rise and late decay phase of this event: the light curve is shown in Fig. 1, lower panel. Shutter movements mean that it is not possible to get RHESSI imaging and spectroscopy exactly coincident with the EIS raster times, however the closest-in-time images are produced and are shown in Fig. 13. We return to a discussion of this in Sect. 5.

\section{Hinode EIS data}

During the flare, EIS was running the observing study HH_AR+FLR_RAS_H01. This is a fast raster mode which scans east to west over a $240^{\prime \prime}$ by $240^{\prime \prime}$ region in $10^{\prime \prime}$ steps using the 1 " slit. An exposure time of $5 \mathrm{~s}$ is used, so the total raster time is then 150s which keeps good temporal information at the expense of some spatial resolution. A wide temperature range from $\log T_{\max } 4.7-7.2(\mathrm{~K})$ is covered by the 17 available emission lines; ideal for the study of dynamic behavior in the impulsive phase.
Table 1. Emission lines used in the analysis.

\begin{tabular}{|c|c|c|c|}
\hline Line & Wavelength $(\AA)$ & $\log T_{\max }(K)$ & Note \\
\hline He II & 256.320 & 4.7 & Si $x, F e$ XII and Fe xIII \\
\hline Si VII & 275.350 & 5.8 & Poor signal \\
\hline Fe VIII & 185.210 & 5.8 & Ni XVI \\
\hline $\mathrm{Fe} x$ & 184.540 & 6.0 & $\ldots$ \\
\hline $\mathrm{Fe} \mathrm{xI}$ & 188.230 & 6.0 & Self blend \\
\hline $\mathrm{Fe} \mathrm{xII}^{*}$ & $186.854+186.887$ & 6.1 & $n_{\mathrm{e}}$ pair \\
\hline Fe xII & 195.120 & 6.1 & $n_{\mathrm{e}}$ pair \\
\hline Fe XIII* & $203.797+203.828$ & 6.2 & $n_{\mathrm{e}}$ pair \\
\hline Fe хIII & 202.040 & 6.2 & $n_{\mathrm{e}}$ pair \\
\hline Fe xiv* & 264.780 & 6.3 & $n_{\mathrm{e}}$ pair \\
\hline Fe xIV & 274.200 & 6.3 & $n_{\mathrm{e}}$ pair \\
\hline $\mathrm{Fe} x \mathrm{v}$ & 284.160 & 6.3 & $\ldots$ \\
\hline Fe xvI & 262.980 & 6.4 & $\ldots$ \\
\hline Ca xvII & 192.820 & 6.7 & $\mathrm{O} v$ and $\mathrm{Fe} \mathrm{xI}$ \\
\hline Fe XxIII & 263.760 & 7.1 & Peak only \\
\hline Fe xxiv & 192.023 & 7.2 & $\mathrm{Fe} \mathrm{XI}$ and $\mathrm{Fe}$ VIII \\
\hline Fe xxiv & 255.100 & 7.2 & $\mathrm{~S} x$ and $\mathrm{Fe} x \mathrm{x} I I$ \\
\hline
\end{tabular}

Notes. The emission lines marked with * are excited from a metastable level.

The raw EIS data were calibrated and cleaned by the SolarSoft routine EIS_PREP. Gaussian fits of the data were made for a selection of spectral lines at different heights along with pairs in Fe XII, Fe XIII and Fe xIV used for density diagnostics. Care was needed in analysing lines involved in density diagnostics as there are a number of blends present. From information given in Young et al. (2007) and Brown et al. (2008) we have attempted to remove the noticeable contributions, details of all the lines discussed are given in Table 1 .

Fe XII $186.88 \AA$ is in fact a blend of two Fe XII lines, $186.854 \AA$ and $186.887 \AA$. For our density diagnostics we will use the sum of both lines referred to as $186.88 \AA$. A small S XI blend at $186.84 \AA$ is reported but from the available wavelength windows this potential blend could not be directly quantified but the ratio of intensity to $195.12 \AA$ is so large that it may not have a significant effect on any density diagnostics. Fe XIII $203.82 \AA$ is more problematic with a significant blend in the red wing identified as Fe XII $203.72 \AA$. This has been removed by using a double Gaussian fit to pick out the smaller Fe XII component from the main Fe xIII line. Fe XIII 203.82 $\AA$ is itself a blend of two Fe XIII lines at 203.828 $\AA$ and 203.797 $\AA$ and again the total of both will be used in density diagnostics.

Fe xIV $274.20 \AA$ has a blend with Si vII $274.18 \AA$, the ratio of Si VII $274.18 / 275.35$ is seen to be no more than 0.25 , so by fitting 
the available Si vII $275.35 \AA$ line, the blend of Si vII $274.18 \AA$ with Fe XIV $274.20 \AA$ can be safely removed.

Not all of the lines present were suitable for analysis. The relatively small nature of the flare meant that good line profiles in hotter flare lines were often completely masked by lower temperature blends, or the signal was too weak to be reliable.

Doppler measurements of Ca XVII $192.820 \AA$ were not used as strong blends of $\mathrm{Fe} \mathrm{XI}$ and $\mathrm{O} \mathrm{v}$ in the wings obscured the centroid position. Fe xxIV 192.023 $\AA$ was strong during the impulsive phase in bright loop regions although an $\mathrm{Fe}$ XI blend in the blue wing makes high speed upflows difficult in places to interpret. Fe XVII $254.87 \AA$ is present in the Fe xxIV $255.100 \AA$ window but can be resolved sufficiently using a double Gaussian fit. The reported weak S x presence does not appear to contribute significantly in footpoint regions, hence there was no requirement for a triple Gaussian fit. Fe XxIII is relatively unblended but only strong enough to observe in a few rasters near the flare peak.

Moving on to cooler lines, Si vII $275.350 \AA$ is unblended but strong signals in places of interest were too intermittent to be of use; velocity shifts when measured were similar to that of He II which is a much stronger line. However, He II $256.32 \AA$ is blended by transitions from Si x $256.37 \AA$, Fe XII $256.41 \AA$ and Fe XIII $256.42 \AA$. We do not have all the necessary lines available to fully de-blend He II, but justify its use here - albeit with caution - from inspection of the line profiles. The most prominent contribution is in an extended red wing, probably from Si x, which has been removed by using a double Gaussian fit. Finally, we have omitted Fe xI due the difficulty in resolving the line pair to obtain accurate Doppler shifts; Fe $\mathrm{x}$ is similar in temperature with a cleaner profile.

Any calculation of velocity shift depends on a good definition of a rest wavelength. This is difficult in active regions. A useful technique of determining the rest wavelength (Milligan 2008 ) is to use a small quiet area near the region as a guide. For each raster, the average centroid position of a $10^{\prime \prime}$ by $100^{\prime \prime}$ quiescent region between $\left[-500^{\prime \prime},-240^{\prime \prime}\right]$ and $\left[-400^{\prime \prime},-230^{\prime \prime}\right]$ was used to calibrate the velocity shifts. The area is free of active region moss, and the coronal loops seen do not contribute much to the average spectral profile which was checked in each raster for anomalous shifts or large intensity variations. EIS suffers from two instrumental variations in the position of line centroids, an offset from the alignment of the CCD and spectrometer slit, and a shift due to the change in temperature in the spacecrafts orbit. The fitted rasters were also corrected for these using standard Solarsoft routines.

During these rasters the EIS spectrograph was slightly out of focus (Warren et al. 2008), as a result there is a significant spatial offset apparent between wavelengths using the different CCDs, sometimes more than 10 pixels in the N-S direction, and around 2 pixels in the E-W direction. In addition to this, the EIS pointing drifts over time, and since our analysis tracks relatively small features - only a couple of pixels across - in a number of wavelengths and over time, we have cross-correlated the raw intensity data prior to fitting to correct for these drifts.

A binary map of the region was produced based on a threshold intensity (set at some percentage of the maximum brightness of each image) and the drift from image to image calculated using the 2D cross-correlation. Drifts of up to $\sim 5^{\prime \prime}$ were found between EIS raster images, and the EIS pointings updated - relative to the first raster - accordingly. Based on this process, and some manual adjustment, we estimate that the EIS raster images are co-aligned within $1.5^{\prime \prime}$ in $x$ and $1.5^{\prime \prime}$ in $y$.

\section{Hinode EIS plasma diagnostics}

The available wavelength windows allowed density diagnostics to be carried out for Fe XII $\lambda \lambda 186.880 / 195.120$, Fe XIII $\lambda \lambda 203.820 / 202.040$ and Fe XIV $\lambda \lambda 264.780 / 274.200$ (see Young et al. 2007). These diagnostics are based on the ratio of two spectral lines, one of which is excited from the ground level and the other from a metastable level, the population of which depends strongly on the local electron density. The ratio of these two lines is sensitive to the electron density in the region where they are formed, and comparison of observed line ratios with theoretical predictions allows the plasma electron density to be deduced. We use the atomic database CHIANTI Dere et al. (2009). The calculations assume ionisation equilibrium and Maxwellian electron distributions.

Five rasters were chosen to best display the evolution of the flare. These are shown in Figs. 4-6. The rasters shown correspond to the centre of each TRACE $171 \AA$ image in Fig. 2. The morphology of the region in these iron lines is similar to that seen in TRACE. Note that in the fourth raster shown there is a drop in intensity towards the left-hand edge. This occurs because Hinode passed into EUV eclipse. However this occurs far enough after the time at which the slit was on the the centre region of interest not to affect our results. This fourth raster shown, starting at 16:09:03 UT will have finished its scan by around 16:11:33 UT, covering the maximum seen in RHESSI at 6-12 keV (Fig. 1). Without any subsequent rasters until 16:38:12 UT this is the best picture we have of the flare peak.

\subsection{Intensity, density and velocities across the region}

The derived intensities, velocity shifts, and densities for each wavelength are shown in Figs. 4-6. It should be noted that because of the sparse raster run, all of the EIS rasters are stretched by 10 pixels in the $X$ direction using the congrid method in IDL. This maintains the correct aspect ratio when making comparisons with other images but does not impose any extra spatial information.

In Figs. 4-6 we show the intensity in both of the spectral lines which are used in the diagnostic. The top row shows the transition excited from the metastable level and the row underneath shows the other transition, excited from the ground level. As is particularly evident in the third Fe XIII and Fe XIV rasters, in some locations the emission involving the transition from the metastable level is increased, as it should be when higher densities are present. Emission in the other line remains relatively unchanged. This indicates a change in the electron density at the formation temperature of these lines, but without a substantial change in the temperature (i.e. the fraction of the particular Fe ion). All three ions shown brighten in similar locations; starting with localised enhancements leading to extended ribbon structures, with the northern areas leading the southern by a few minutes. The situation is supported by a similar picture in the lower temperature lines He II and Si vII, which appear more sensitive to early impulsive phase activity.

The electron density calculated from the diagnostic ratio is shown in the bottom row. A density enhancement is present, and has a similar structure, in all of the diagnostic ratios we have calculated. A few small, perhaps footpoint-related, enhancements are formed prior to the impulsive phase in the raster starting at 15:55:47 UT. Those situated north of the dark filament structure in the centre of the EIS field of view form first slightly preceding those in the south. They remain to become part of two high 

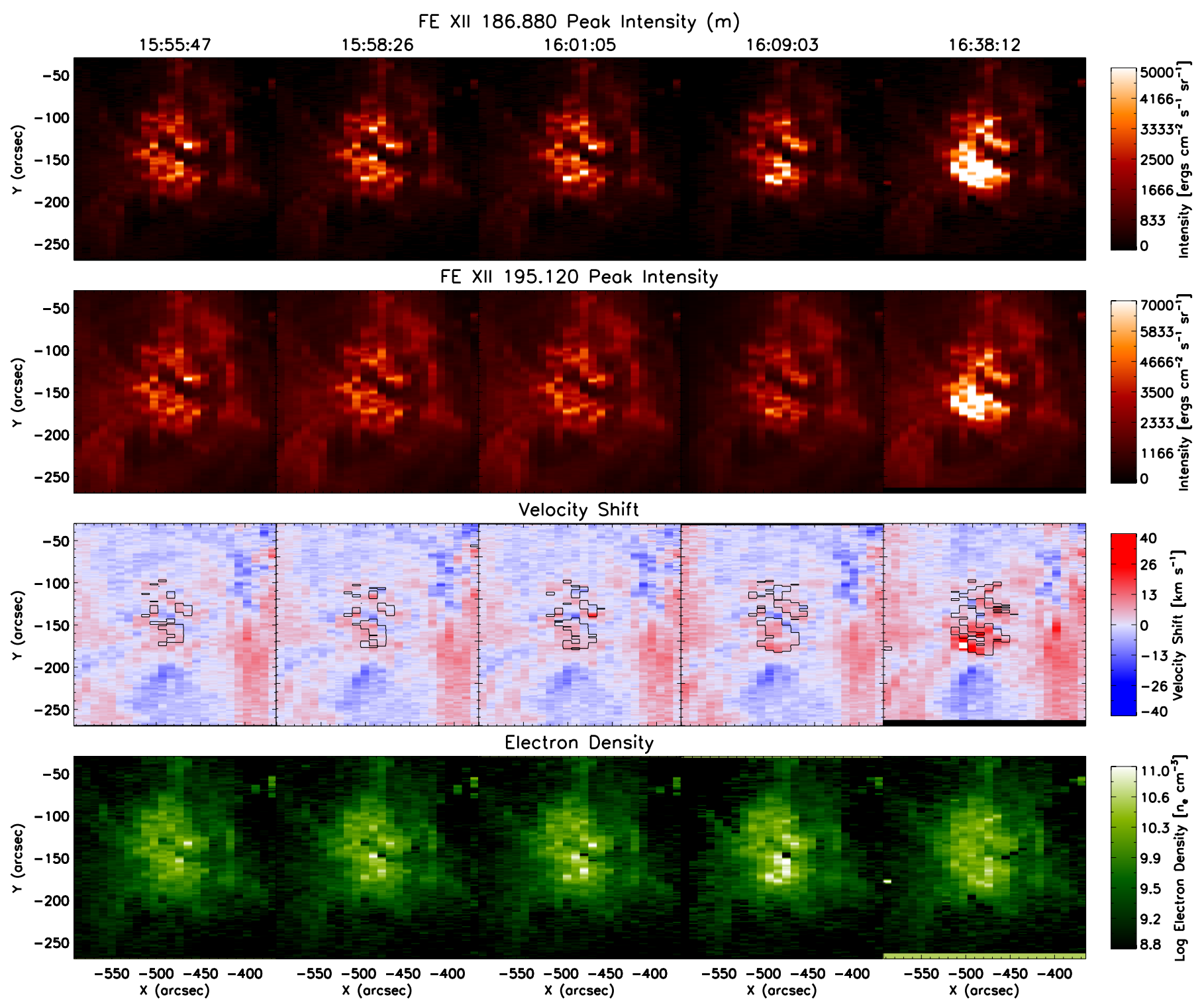

Fig. 4. Fitted rasters for Fe xII. The top and second row show the intensities in each of the lines used in forming the diagnostic, with the upper one being produced by excitation from the metastable (density sensitive) level. Velocity maps are produced using the $195.120 \AA$ line and overlaid with density contours at $\log n_{\mathrm{e}}=10.2$. The first four columns correspond to the impulsive phase, with the fourth column at the observed peak.

density areas on either side of the filament, matching the pattern of the EUV ribbons seen in TRACE at 16:10:12 UT. In the final post-flare raster, both lines in the diagnostic pair show enhancements, as well as a density increase. Bright post-flare ribbons, also seen at 16:38 UT in TRACE, are very clear in the Fe XIII density map but less obvious in Fe XII, possibly as the density in the surrounding region is higher. A bright arc linking to the north area can be seen faintly. The southern post-flare ribbon is fainter again in the Fe XIV density map but the same section becomes more visible slightly later than the other lines.

The third row of Figs. 4-6 shows the line-of-sight velocity shift relative to the "rest" wavelength. Over-plotted are black contours showing the locations of density enhancements, highlighting the overlap with velocity shifts. Small velocities, on the order of 20-25 $\mathrm{km} \mathrm{s}^{-1}$ maximum are seen. Though we do not show all of the raster images, blueshifts or upflows are seen predominantly in lines from ionisation stages above Fe XIII and redshifts or downflows seen from lower ionisation lines (see Figs. 10-12), as was found by Milligan \& Dennis (2009). In the decay phase, strong downflows and density enhancements occur in all lines as material cools and falls. Note also the blueshifted region to the north-west corresponding to the fan of loops seen in the TRACE images. This may be the same phenomenon as the large scale flows from the edge of active regions reported by Harra et al. (2008).

\subsection{High velocity flows}

Higher temperature lines, hotter than the density diagnostic pairs, also display strong upflows. Figure 7 displays a selection of velocity maps from the raster at 16:01:05 UT where the flows in most lines reach their maximum velocity (as seen later in Figs. 10 to 12 ).

Fe XVI at $\log T_{\max }=6.4$ is a good diagnostic of dynamic motions with no blends and a strong signal. It is the only line where we could perform a reliable double Gaussian fit to search for high velocity components at the footpoints. In rasters nearing the flare peak, an asymmetric enhancement in the blue-wing of the line profile was present. We used the standard EIS fitting routines with a two-Gaussian template to produce the images 

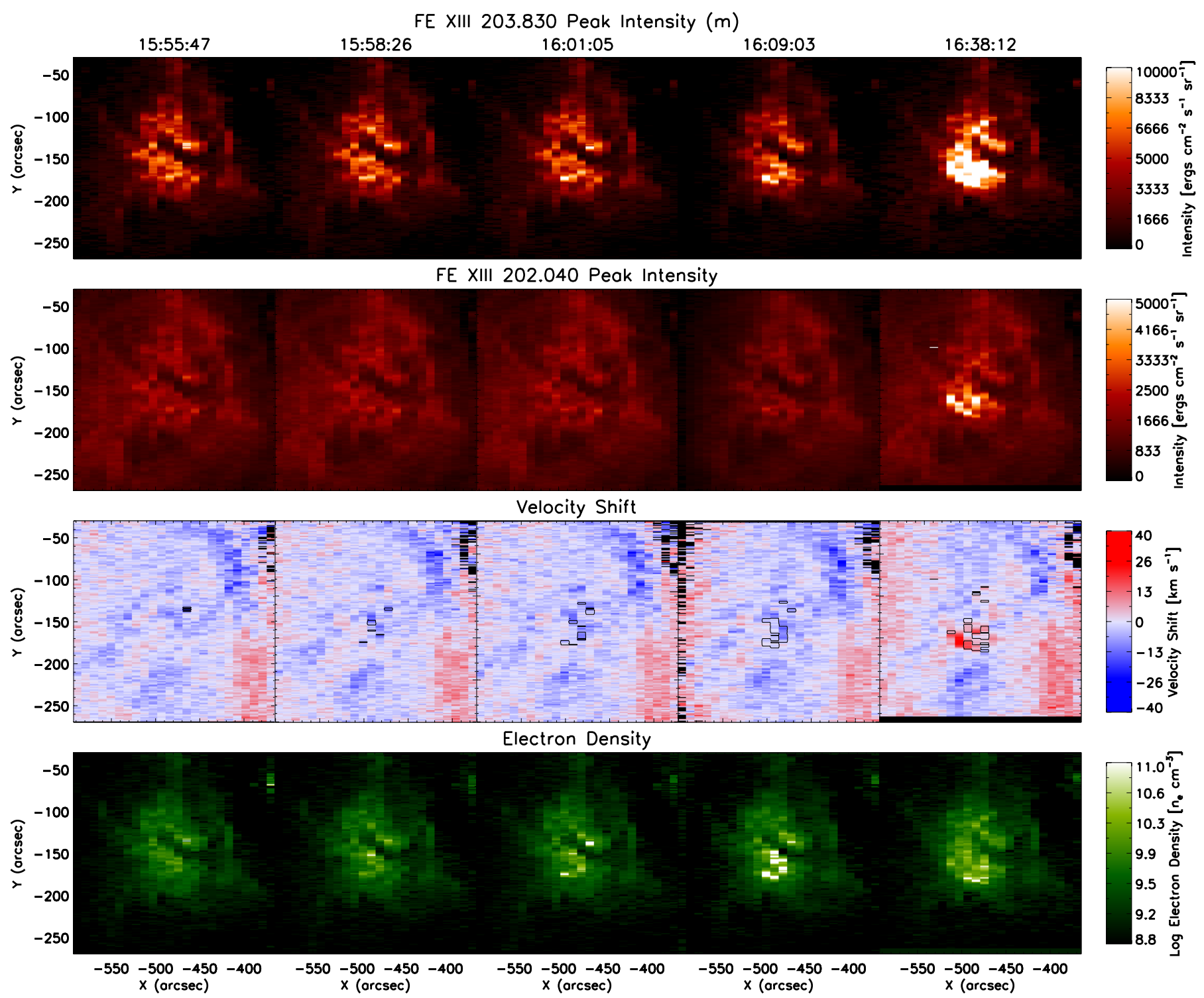

Fig. 5. As in Fig. 4 but for the Fe XIII density diagnostic pair. Velocity maps are produced using the $202.04 \AA$ line and overlaid with density contours at $\log n_{\mathrm{e}}=10.2$.

found in Fig. 7. We find the central Gaussian component is blueshifted in the flare ribbon regions up to around $40 \mathrm{~km} \mathrm{~s}^{-1}$. At the same locations the second Gaussian component is found to be strongest in intensity along with blue-shifts of 110 to $140 \mathrm{~km} \mathrm{~s}^{-1}$.

Double-Gaussian fitting by nature can be susceptible to creating acceptable but un-physical fits to data. At three positions of interest (marked in Fig. 9 and discussed in Sect. 4.3) we look at the line profiles of Fe XVI more carefully. The signal is first binned with 2 pixels above and below to ensure a high SNR. Next, using the R-B Asymmetry method found in De Pontieu et al. (2009), the position of maximum asymmetry in the line was found - where asymmetry is defined as the deviation from a single fitted Gaussian profile. The second Gaussian component was then initiated at this location. Gaussian widths were only restricted by a lower limit equal to the instrumental width. Profiles at the different positions for a selection of rasters are found in Fig. 8.

Taking the first row at Position 0, again for times around 16:01 UT, the asymmetry is clearly notable. Velocity shifts inferred from the second Gaussian range between 100$140 \mathrm{~km} \mathrm{~s}^{-1}$, however the contribution of this component to the whole distribution is strongest at 15:55 UT and varies throughout. Moving to the bottom row of plots, Position 1 exhibits a strong asymmetry but slower upflows at $42 \mathrm{~km} \mathrm{~s}^{-1}$, at Position 2 the asymmetry is much weaker but an upflow can still be detected at $115 \mathrm{~km} \mathrm{~s}^{-1}$. Surprisingly, the centre component is still found to be blue shifted when adding a second component to the fit, and quite strongly so at 16:01 UT Position 0. This does not fit a situation where high speed upflows found at flare footpoints coincide with stationary background emission (Del Zanna et al. 2011).

Given the profiles in Fig. 8, and knowledge that the fits were based on the position of maximum asymmetry, we can be reasonably confident in saying there is a significant amount of plasma at $\log T_{\max }=6.4$ moving between 100 and $140 \mathrm{~km} \mathrm{~s}^{-1}$ at Position 0 and a small contribution of around $110 \mathrm{~km} \mathrm{~s}^{-1}$ at Position 2.

Significant blue shifts were found in the Fe xxIv lines. The $255.100 \AA$ line has good signal across the region and displays shifts of $40-60 \mathrm{~km} \mathrm{~s}^{-1}$, although a more thorough double component analysis could not be carried out because of the close proximity to the Fe XvII line. High speed flows were also seen 
D. R. Graham et al.: Hinode/EIS flare density diagnostics
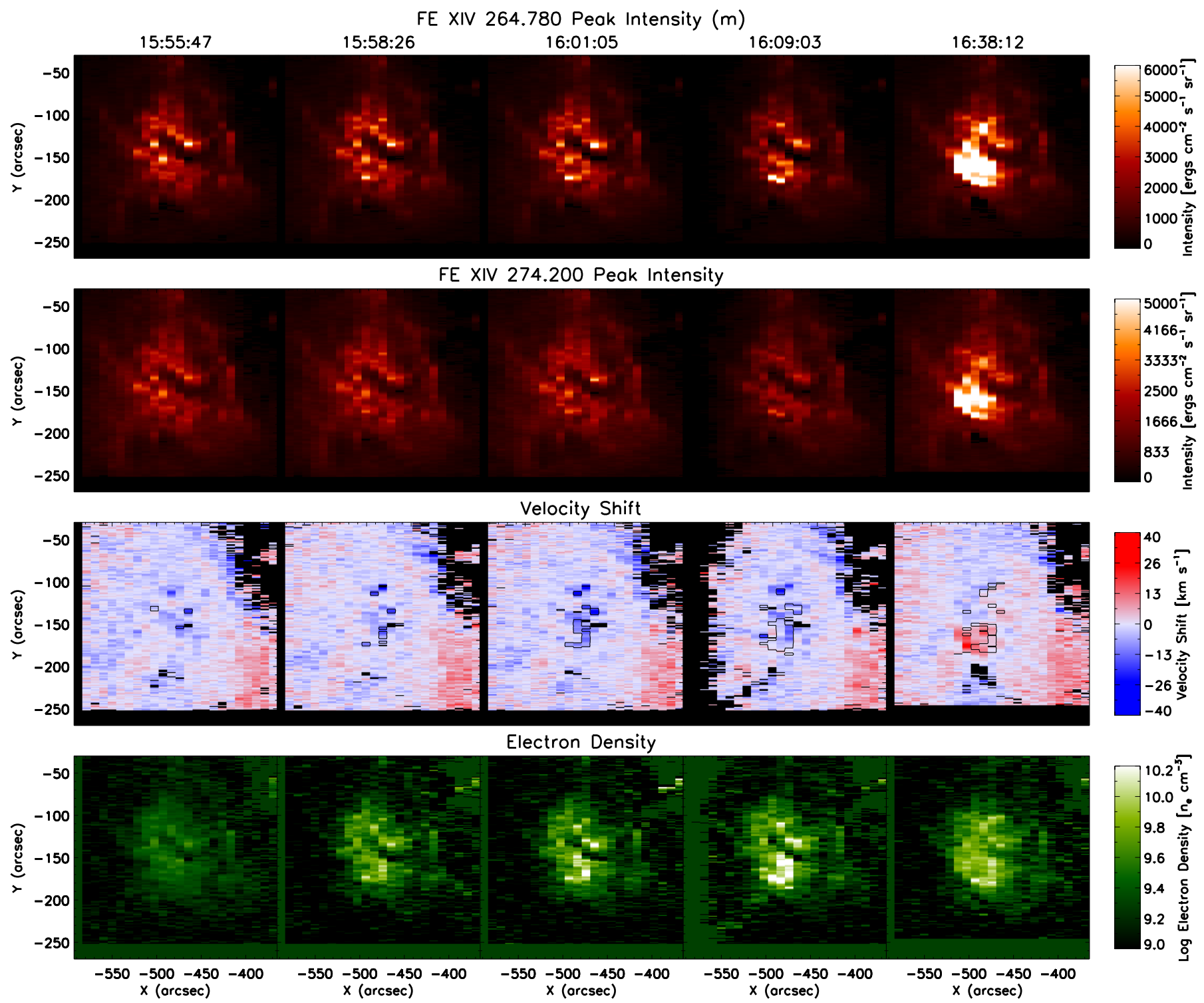

Fig. 6. As in Fig. 4 but for the Fe xIv density diagnostic pair. Velocity maps are produced using the $264.78 \AA$ line and overlaid with density contours at $\log n_{\mathrm{e}}=9.9$.
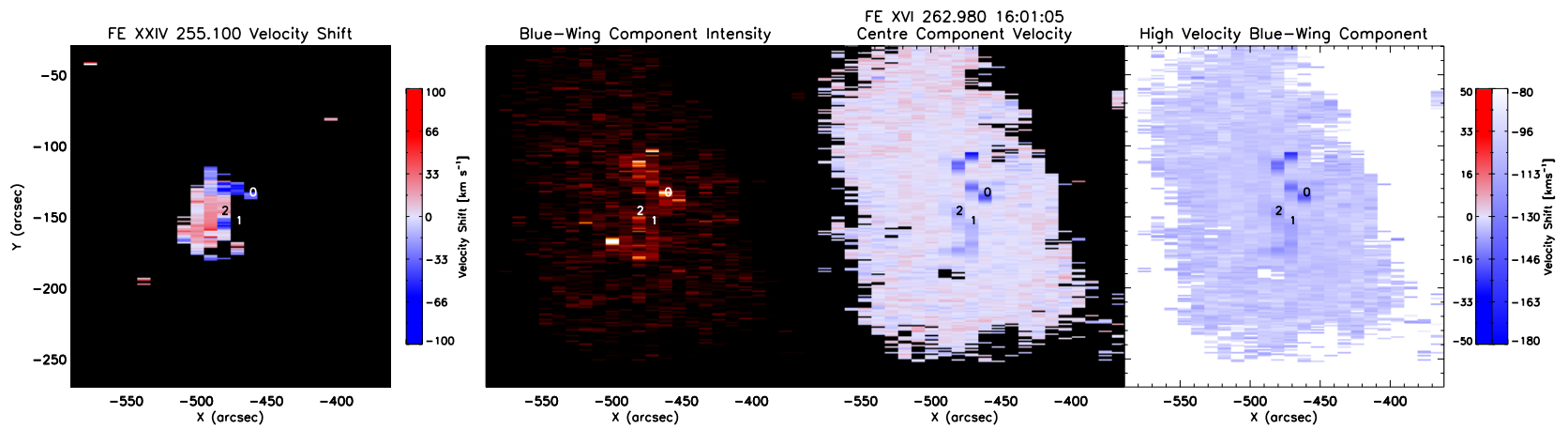

Fig. 7. A high temperature line analysis for Fe xxIv (left hand image) and Fe xvI (right hand images). Fe xxvI velocity shifts derived from a single Gaussian fit are are shown in the left hand image while images from a double Gaussian fit of Fe xvi are shown on the right. From left to right: the intensity of the second Gaussian component, the velocity shift of the centre component and velocity shift of the second component found in the blue-wing - note the corresponding velocity ranges for each image. The positions marked are identical to those shown in Fig. 9 and Sect. 4.3 .

in Fe xxIv 192.023 $\AA$ which brightened in rasters starting from $15: 55: 47$, with emission forming a loop just to the east of the southern "U" shaped ribbon. This line is blended with Fe XI $191.808 \AA$, but in the loop the Fe xxIV signal is strong and furthermore there is no sign of enhanced emission in the Fe $\mathrm{xI}$ $188.230 \AA$ line observed also at this location. Upflows of around $200 \mathrm{~km} \mathrm{~s}^{-1}$ were found at the southern end of the loop at 15:55:47. However, these flows are only present in the early 

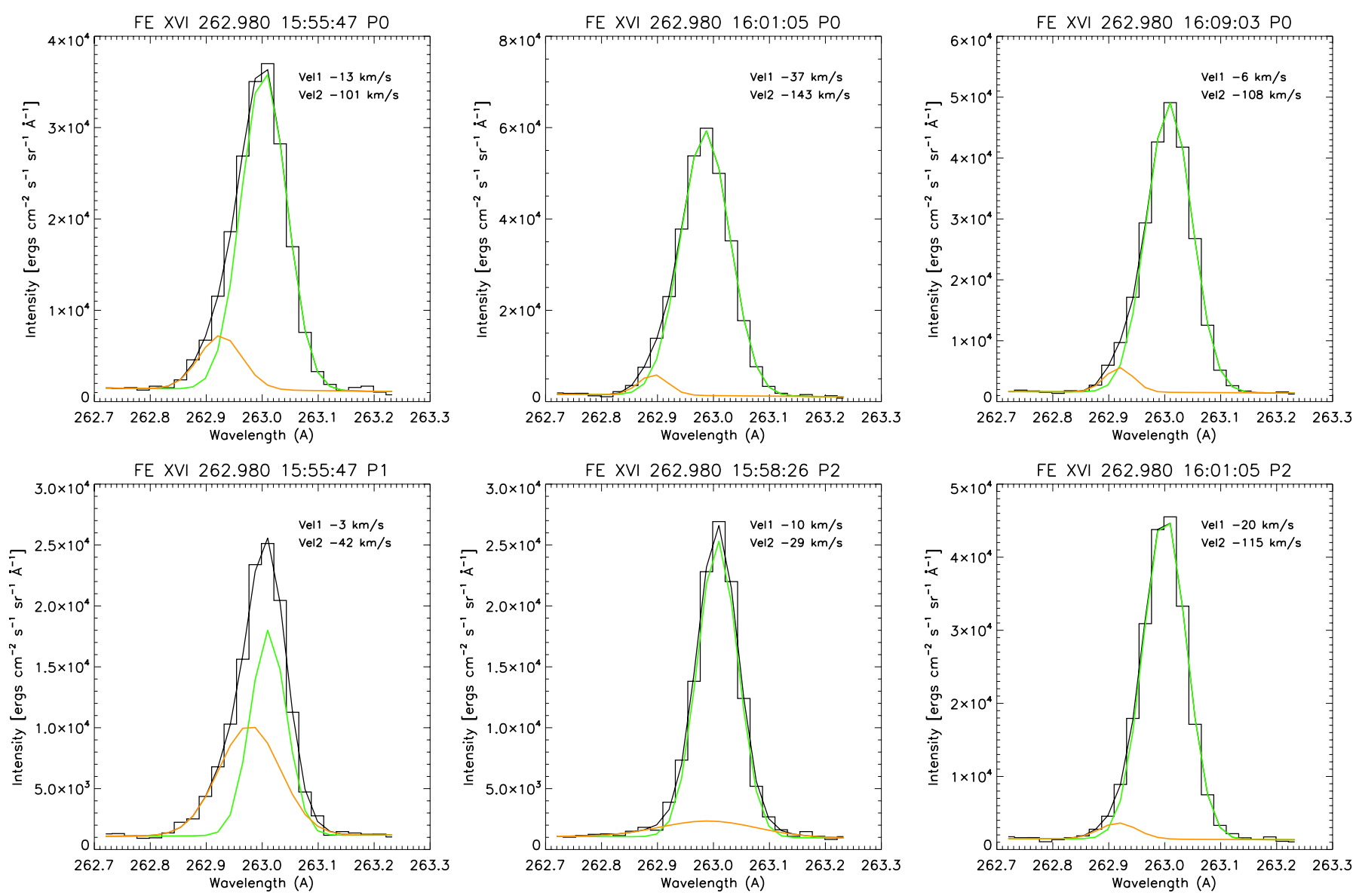

Fig. 8. Fe xvi double component fits at positions shown in Fig. 9. The two Gaussian components of the fit are marked in green and orange, the total fit by the solid black line, and the data by the stepped black line. Velocities of both components (Vell: green, Vel2: orange) are derived from a common reference wavelength for each raster. Plots on the top row show the evolution of the enhanced blue wing component at Position 0 during the impulsive phase of the flare. In the other two positions, shown on the bottom row, the enhancement is only prevalent early on in Position 1 and only just noticeable at Position 2.

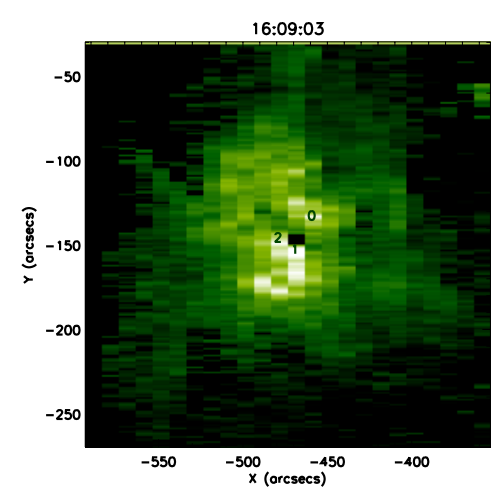

Fig. 9. Pixels chosen for further analysis at positions, displayed on an electron density image from the flare peak.

rasters (before the main HXR peaks), and do not appear to be related to any brightening in cooler lines, nor are they near the footpoints seen in TRACE. We do not have RHESSI images for this time.

\subsection{Examination of selected pixels}

The character of points of strong intensity or density appear to vary quite widely across the region, although for the most part remain bounded by the flare ribbons. We select three points for further analysis, shown in Fig. 9, chosen on the basis of their having large density enhancements or interesting flow patterns. Position 0 marks the earliest footpoint brightening in the region found at the end of the northern ribbon, the highest electron density is found at Position 1, and Position 2 marks the first enhancement found in the southern ribbon. The time evolution of plasma parameters from these locations are shown in Figs. 10 to 12. For each position, the fit parameters of the pixel were averaged with the pixels above and below.

Oppositely-directed flows in Position 0 are immediately apparent from Fig. 10. Fe XVI, Fe XIV and Fe XIII are blueshifted almost simultaneously with redshifts appearing in Fe XII, Fe x, and He II. Blueshifts increase with the line temperature, reaching almost $40 \mathrm{~km} \mathrm{~s}^{-1}$ in the centre component of Fe XVI. Redshifts are present on the order of $10 \mathrm{~km} \mathrm{~s}^{-1}$ in lower temperature Fe lines, however He II only dips slightly compared to pre-flare levels. From Sect. 4.2 we see Fe XxIV is also blueshifted by $35 \mathrm{~km} \mathrm{~s}^{-1}$ at 16:01 UT but not by any more than Fe xIV. Stronger flows of nearer $60 \mathrm{~km} \mathrm{~s}^{-1}$ are seen in Fe xxIv but further to the east. As mentioned previously, higher velocity blue-wing components in this line could be present but here are masked by blends. Upflows in Fe XVI of up to $140 \mathrm{~km} \mathrm{~s}^{-1}$ are however found in the enhanced blue-wing component (see Sect. 4.2), indicative of explosive evaporative behaviour. From Culhane et al. (2007), EIS can measure velocity shifts to within $\pm 5 \mathrm{~km} \mathrm{~s}^{-1}$, but since we cannot be certain that our chosen "rest" wavelength corresponds to zero velocity, only relative velocity shifts can be discussed 
D. R. Graham et al.: Hinode/EIS flare density diagnostics

Position 0
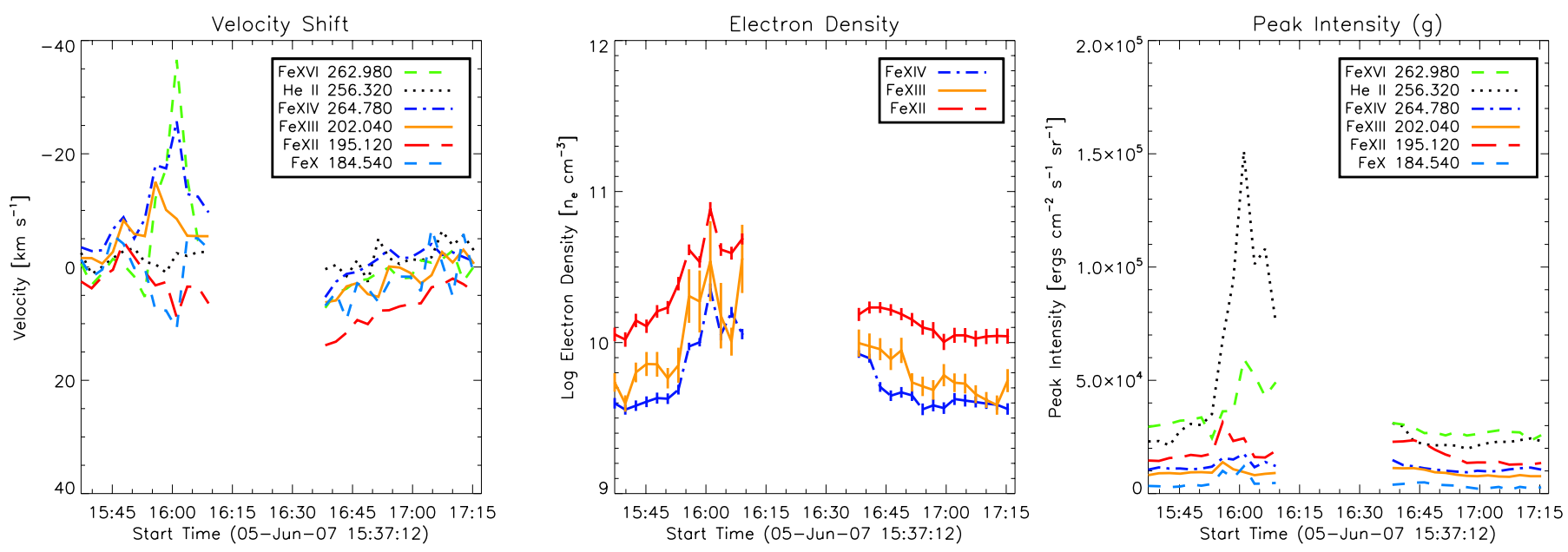

Fig. 10. Time evolution of plasma parameters in the bright region labelled "0" in Fig. 9. From left to right, the panels show the line-of-sight velocity shift in five ionisation stages of iron plus one helium, the density variations, and the intensity including the components of the density diagnostic excited from the ground state. Velocities for Fe xvI are derived from the centre component of the double fits in Sect. 4.2.

Position 1
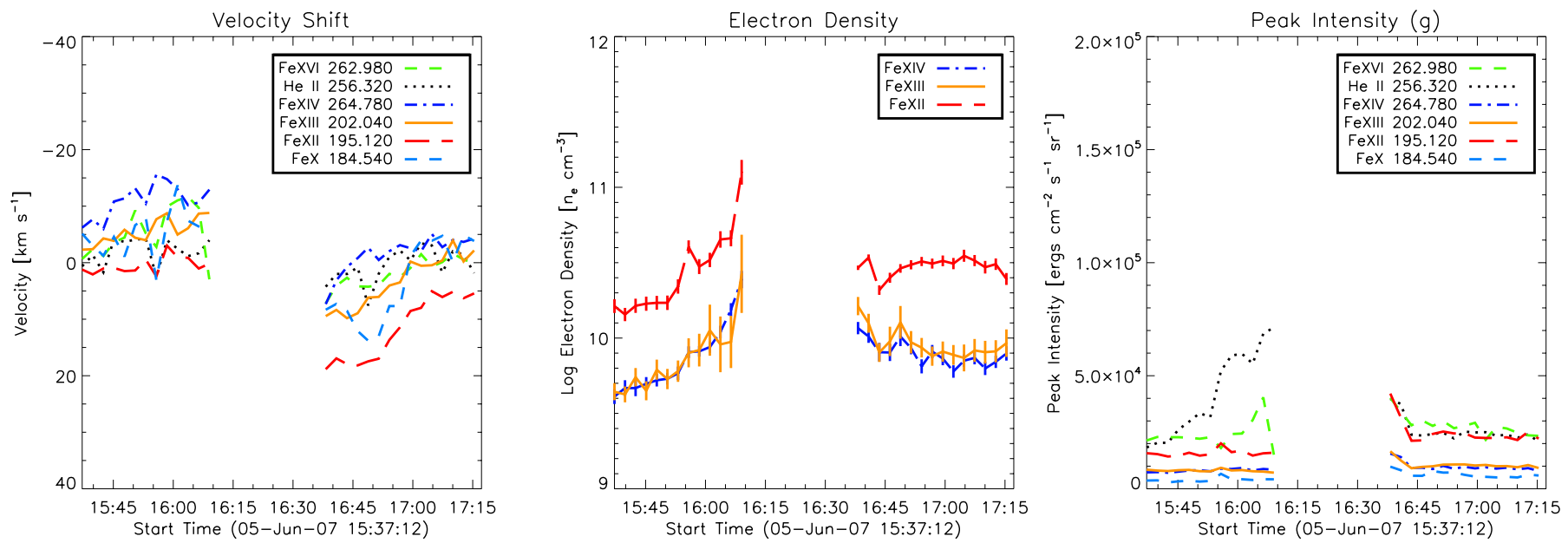

Fig. 11. As in Fig. 10 but showing parameters for position "1".

Position 2
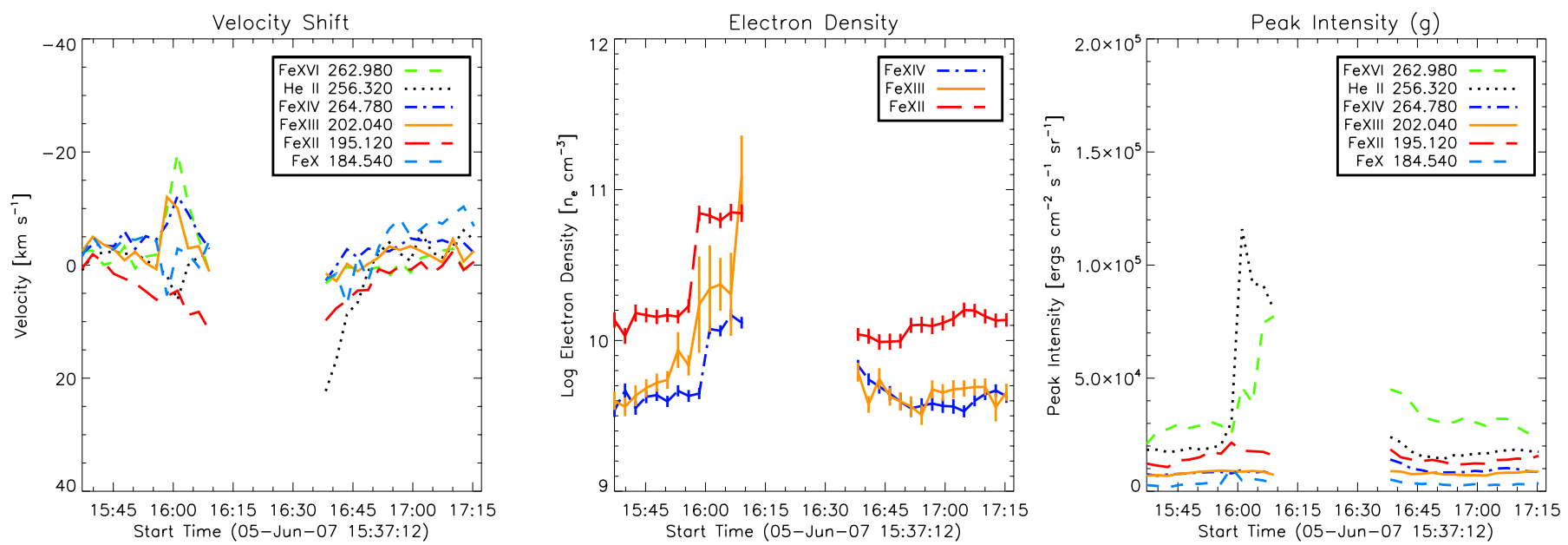

Fig. 12. As in Fig. 10 but showing parameters for position "2". 
with any confidence. Also this does not apply in the same way to high velocity components derived from a double Gaussian fit.

As the velocity shifts peak at 16:01 UT, the electron number density peaks in all three diagnostics at $\log n_{\mathrm{e}}=10.2\left(n_{\mathrm{e}}\right.$ in $\mathrm{cm}^{-3}$ ) in Fe XIV and $\log n_{\mathrm{e}}=10.9$ in Fe XII. The Fe XIII diagnostic used saturates about $\log n_{\mathrm{e}}=11.3$ but due to problems in the atomic physics can not be trusted above $\log n_{\mathrm{e}}=10.3$ (Young et al. 2009). Although it appears to follow the behaviour of the other diagnostics reasonably well, it fluctuates more at high densities. We have where possible interpolated the upper and lower limits of the measured intensity ratios onto the density curve, returning the possible range of electron density.

The intensities of the spectral lines excited from the ground state - as opposed to the metastable state - show only small changes throughout the impulsive phase observations between 15:55 UT and 16:05 UT. Changes are most notable and sudden in Fe XII. As discussed in Sect. 4.1, the relative stability of these lines suggests that the Fe ionisation fractions, and hence the temperature, of the emitting plasma are not changing significantly.

Position 1 (Fig. 11) located on the southern side of the filament presents a different picture. The electron density here is the highest seen during the flare, rising to $\log n_{\mathrm{e}}=10.3$ in Fe XIII and Fe xIV, and to $\log n_{\mathrm{e}}=11$ in Fe XII. Hotter lines while blueshifted do not display the same well defined increases as Position 0. He II and Fe XII slightly redshift at 16:01 UT but not significantly. There is some evidence of a high speed upflow present in Fe XVI of $42 \mathrm{~km} \mathrm{~s}^{-1}$ (Fig. 8) at 15:55 UT but the center component is not significantly shifted at $3 \mathrm{~km} \mathrm{~s}^{-1}$. Fe xxIV also has no strong signal here.

Finally, Position 2 (Fig. 12) is where a density enhancement in the southern ribbon first arises, eventually forming the " $U$ " shaped density enhancement south of the filament. Measured densities do not appear to rise until after 15:55 UT where all three diagnostics suddenly jump to $\log n_{\mathrm{e}}=10.2$ in Fe xIV and $\log n_{\mathrm{e}}=10.8$ in Fe XII; possibly explained by the scanning slit passing over the edge of the flare kernel which may be smaller than the 10" raster spacing. Again there are significant upflows in lines hotter than Fe XIII but only to speeds of around half that of Position 0. Simultaneous redshifts are present at this position in $\mathrm{Fe} \mathrm{x}$ and $\mathrm{He}$ II on the order of $5-10 \mathrm{~km} \mathrm{~s}^{-1}$. Although weaker than Position 0, here we see Fe xvi blue-wing flows of $115 \mathrm{~km} \mathrm{~s}^{-1}$ and surprisingly a red shift in Fe XxIV of $12 \mathrm{~km} \mathrm{~s}^{-1}$. The flows in Fe xxIV do not appear to match those of lower temperature lines; blue-shifted regions in Fe XxIV are found just to the south of Position 2, as is a stronger flow found to the east of Position 0. It is unlikely that this is an image correlation issue as both Fe XVI and Fe XxVI use the same long wavelength CCD. In the same rasters Fe XxIII also blueshifts in these areas by around $50 \mathrm{~km} \mathrm{~s}^{-1}$. The line profiles deviate strongly from a symmetrical Gaussian, and if Fe XxIII is believed to be unblended, represent large high speed components. Unfortunately the signal is very weak, around $20 \%$ of Fe xvI and xxvi, and multiple Gaussian fitting is found to be unreliable in this case.

\section{RHESSI data analysis}

The times for analysis in RHESSI were chosen to match the two peaks in emission whilst avoiding periods when the attenuating shutters were out, which suffered badly from pulse-pileup. RHESSI images were produced using detectors 3, 4, 5, 6 and 8 with the CLEAN algorithm. The spectral analysis was done with detector 1 data as it had the best energy resolution and lowest background during this pre-anneal time (Hannah et al. 2010). OSPEX was used to fit a thermal plus thick-target non-thermal

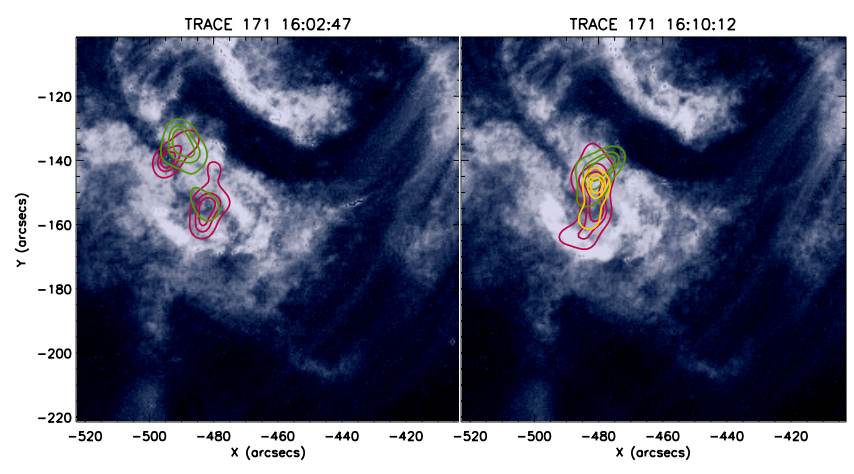

Fig. 13. Impulsive phase TRACE 171 images with RHESSI 60\%, 80\% and $90 \%$ intensity contours overplotted in 6-12, 12-18 and 18-25 keV energies (magenta, green and yellow respectively).

model to this data, with an eclipse background (in A1) subtracted.

From these, the energetics of the electron beam can be estimated (see Sect. 6), and the location of the footpoints. The pointing difference between TRACE and RHESSI is unknown, but it is possible to align images via SOHO/MDI since its pointing is well known, as is RHESSI's. SOHO/MDI continuum images and TRACE WL were cross correlated to find the residual pointing difference, which is then used to move the TRACE images relative to RHESSI. MDI continuum is only available a few times a day, in this case at 12:29 UT, and was rotated using the SSW DROT_MAP software to match the TRACE WL at 15:31 UT then corrected for the Earth parallax shift. Further drift between TRACE $171 \AA$ images was corrected for by tracking the movement of stable bright points north of the filament.

After these corrections, we estimate the pointing of TRACE with respect to RHESSI to be within $1.5^{\prime \prime}$ in each direction. The results for two times are shown in Fig. 13. The RHESSI contours lie on top of bright TRACE $171 \AA$ regions which we associate with chromospheric emission. Surprisingly, there is no evidence for a HXR footpoint at Position 0 (Fig. 10) north of the small filament, yet of the three positions studied this shows the most convincing chromospheric evaporation signatures. The HXR sources are in the southern ribbon, where evaporation signatures are not so strong. Even at lower values of the RHESSI contours, no HXR sources at Position 0 can be found. An offset of $\sim 20^{\prime \prime}$ between the TRACE and RHESSI images would be necessary to align RHESSI sources with Position 0, and we believe that such an error in relative pointing is not reasonable given our co-alignment process, and given the fact that the shape of the HXR emission matches well with the TRACE emission. If electron beam energy input is driving evaporation here, then the beam must be relatively weak in comparison to other locations.

Figure 14 shows the spatially integrated photon spectrum corresponding to both images in Fig. 13. Each time step is fitted with two models; a thick target with a single thermal component, and with a pair of thermal components. An elevated background, due to degraded detector performance, in this event means that the non-thermal component is not particularly clear, but in the spectrum presented for 16:02:40 UT and 16:09:52 UT the thermal fit (red line) does break away noticeably from the data above around $12 \mathrm{keV}$. The two thermal components also provide an acceptable fit to the data but again the high background makes it difficult at very high energies, where the high temperature thermal fit (orange line) drops slightly lower than in the non-thermal model (blue line). The fit parameters for these intervals are shown in Table 2, and will be used later in esti- 
D. R. Graham et al.: Hinode/EIS flare density diagnostics

Table 2. Thermal plus thick-target model and double thermal model fit parameters for the RHESSI photon spectrum at 16:02 UT and 16:09 UT.

\begin{tabular}{lcccccccc}
\hline \hline Fit & Time & $\begin{array}{c}E M_{1} \\
\left(10^{48} \mathrm{~cm}^{-3}\right)\end{array}$ & $\begin{array}{c}E M_{2} \\
\left(10^{46} \mathrm{~cm}^{-3}\right)\end{array}$ & $\begin{array}{c}T_{1} \\
M K\end{array}$ & $\begin{array}{c}T_{2} \\
\mathrm{MK}\end{array}$ & $\delta$ & $\begin{array}{c}E_{\mathrm{C}} \\
(\mathrm{keV})\end{array}$ & $\begin{array}{c}N_{\mathrm{e}} \\
\left(10^{35} \mathrm{electrons} \mathrm{s}^{-1}\right)\end{array}$ \\
\hline Double Thermal & $16: 02: 40$ & $0.6 \pm 0.1$ & $4.3 \pm 0.9$ & $15.7 \pm 1.0$ & $27.0 \pm 0.8$ & $\ldots$ & $\ldots$ & $\ldots$ \\
Thermal + Thick Target & $16: 02: 40$ & $6.8 \pm 0.2$ & $\ldots$ & $16.4 \pm 1.1$ & $\ldots$ & $9.3 \pm 0.4$ & $15.6 \pm 0.6$ & $2.7 \pm 1.0$ \\
\hline Double Thermal & $16: 09: 52$ & $1.7 \pm 0.5$ & $8.5 \pm 2.3$ & $14.3 \pm 0.8$ & $25.9 \pm 0.9$ & $\ldots$ & $\ldots$ & $\ldots$ \\
Thermal + Thick Target & $16: 09: 52$ & $1.3 \pm 0.3$ & $\ldots$ & $16.2 \pm 0.6$ & $\ldots$ & $9.7 \pm 0.3$ & $16.5 \pm 0.5$ & $2.6 \pm 0.7$ \\
\hline
\end{tabular}

Notes. $E M$ and $T$ are the emission measure and temperature of each thermal component, $\delta$ is the electron spectral index, $E_{\mathrm{C}}$ the electron low energy cutoff, and $N_{\mathrm{e}}$ the electron number flux.
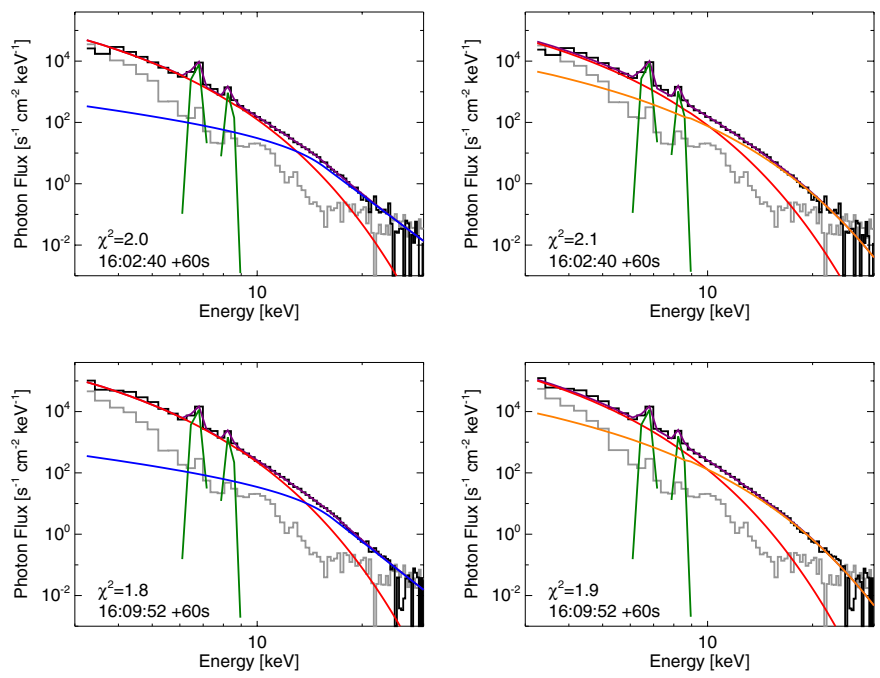

Fig. 14. RHESSI photon spectra at times corresponding to the flare peak (bottom), and the smaller, earlier peak in the 12-25 keV light curve (top). The data for both peaks is fitted with two models; a single thermal component (red) plus thick target model of accelerated electrons (blue), and a pair of thermal components (orange and red).

mating the flare power. Visually, and even from the Chi-Squared values there is little evidence to favour one model over another. Nevertheless, the implications of both are discussed in the next section.

Looking again at the morphology of the RHESSI contours in Fig. 13, the earlier frame appears consistent with hot footpoint emission. Two regions of similar size to the patchy bright regions in TRACE lie in the 6-18 keV energy range. From the spectra, we find these energies are dominated by hot thermal emission at around $16 \mathrm{MK}$. At 16:10 the emission is less localised, possibly outlining the shape of a loop at $6-12 \mathrm{keV}$, yet the concentration at $18-25 \mathrm{keV}$ hints at a footpoint contribution from below. Although the emission does appear loop-like, it lies south of the filament within field thought to be of one polarity so is unlikely to be a loop.

\section{Processes taking place at the flare footpoints}

The observations presented here can be summarised as follows: In the impulsive phase of this flare, energy is deposited into a number of small regions in the chromosphere/lower corona, visible as TRACE ribbons and footpoints. Strong footpoints are also visible in Hinode/XRT, implying plasma at low heights but at temperatures of a few MK. Hinode/EIS Fe line density diagnostics formed at around 1.5 MK show density increases during the early impulsive phase, typically up to a few times $10^{10} \mathrm{~cm}^{-3}$, but since only the more density-sensitive component of the diagnostic pair changes we infer that any variation in tem- perature of the plasma emitting these lines is small. A number of the footpoints show line-of-sight velocity shifts during the impulsive phase - on the order of $20-140 \mathrm{~km} \mathrm{~s}^{-1}$ - at the time of the $n_{\mathrm{e}}$ enhancements, and with a tendency for lines cooler than Fe XIII to be red-shifted and those hotter to be blue-shifted. These are followed in the gradual phase by more substantial redshifts in most lines, notably in images of Fe XII through to Fe xIv, which may be associated with the draining of hot material. The footpoints showing the strongest impulsive phase line shifts (Position 0 in Fig. 9 and regions north of the filament) do not have any detectable RHESSI sources associated with them. Higher speed upflows of around $200 \mathrm{~km} \mathrm{~s}^{-1}$ are found in Fe XxIV to the east of the southern footpoint region, but do not appear to be related to any lower temperature flows or brightenings.

What process is leading to the increased $n_{\mathrm{e}}$ values measured? The most straightforward explanation is to assume that the observed Fe emission is emitted primarily by plasma at the $T_{\max }-$ temperature of maximum ionisation fraction - of the relevant ion, and that during the flare impulsive phase deeper layers of the atmosphere are heated to this temperature. Correspondingly, the dominant emission originates from regions of higher $n_{\mathrm{e}}$, but always at around $T_{\max }$. Although an increase in the $n_{\mathrm{e}}$ of the emitting medium should lead to a higher emissivity for both components of the spectra line, the more density-sensitive one will show a substantially larger increase.

During the flare impulsive phase the atmospheric structure will be changing, although the majority of the velocity shifts observed in this small event are fairly low, the presence of high speed flows in Fe Xvi suggests that there is some dynamic plasma motion here consistent with explosive evaporation. Modelling of gentle evaporation, and of the gentle early phase of flare which later show more explosive evaporation (Fisher et al. 1985a; Allred et al. 2005), shows that the position of the transition region (i.e. the location of rapid increase in atmospheric temperature) moves downwards slightly in the atmosphere and the density just below the transition region increases, roughly consistent with what we see here. However, existing models use input electron spectra that are much harder and typically have a higher cut-off energy than we infer from HXR measurements, so that the majority of collisional energy input occurs deep in the atmosphere. Direct comparisons of our results with these models are therefore difficult.

The diagnostics show that $n_{\mathrm{e}}$ increases typically to a few $\times 10^{10} \mathrm{~cm}^{-3}$ while the high-density sources remain compact (i.e. not loop-like). This places the emission location relatively low in the atmosphere. In a non-flaring (but still active) atmosphere, for example the VAL-E model for bright network (Vernazza et al. 1981) appropriate for the active region locations where the flare occurs, a density of $n_{\mathrm{e}} \sim n_{\mathrm{h}}=4 \times 10^{10} \mathrm{~cm}^{-3}$ occurs at a column depth of $5.8 \times 10^{18} \mathrm{~cm}^{-2}$, where the hydrogen ionisation fraction is approximately equal to unity. In some instances, for example the Fe XII diagnostic at Position $1, n_{\mathrm{e}}$ approaches $10^{11} \mathrm{~cm}^{-3}$, 


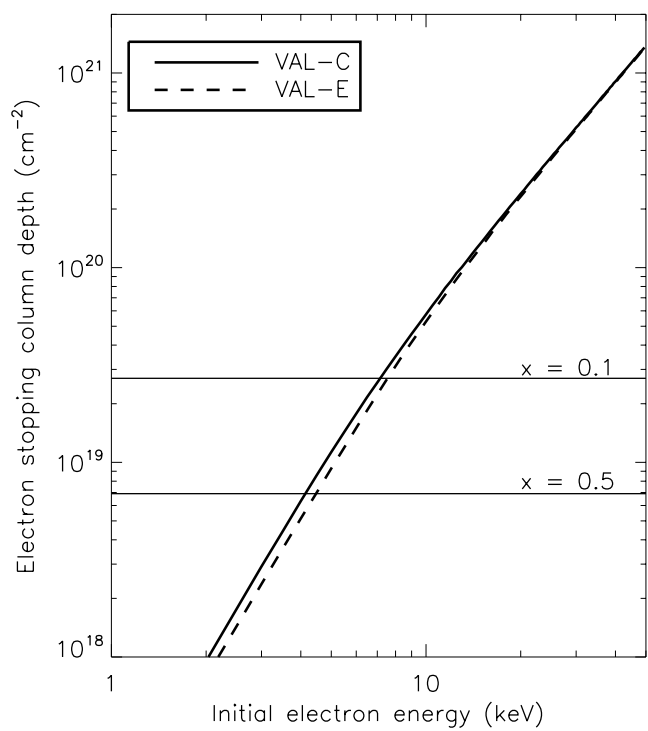

Fig. 15. Stopping column depth as a function of injected electron energy, for the VAL-C and VAL-E model atmospheres, and for model atmospheres with the hydrogen density structure of VAL-C and VAL-E but assuming full ionisation throughout.

which corresponds to regions where ionisation fraction in the VAL-E atmosphere is less than unity. In such cases, one possible scenario is that an electron beam leads to an increase in the observed electron number density due to collisional ionisation, followed by heating. This requires electron beams able to penetrate to regions where ionisation fraction is less than one, but as we show below this is not a demanding condition.

Electron stopping depth. The collisional stopping depth $N_{\text {stop }}$ of an electron of energy $E$ and speed $v$ is found from the following equation (Emslie 1978)

$\frac{\mathrm{d} E}{\mathrm{~d} N}=\frac{-2 \pi e^{4}}{E}\left[x \Lambda+(1-x) \Lambda^{\prime}\right] n v$

where $x$ is the ionisation fraction, $\Lambda$ is the Coulomb logarithm, $\Lambda^{\prime}$ is the associated Coulomb logarithm and $e$ the electronic charge (Emslie 1978). This can be integrated to find the stopping column depth as a function of the initial electron energy. This is shown in Fig. 15 for the VAL-C and VAL-E models. Figure 15 also indicates the approximate locations at which the VAL-E atmosphere is $50 \%$ and $10 \%$ ionised, demonstrating that electrons of energy around $10 \mathrm{keV}$ can penetrate deep into regions of the atmosphere and may increase the hydrogen ionisation fraction.

Electron beam power. We fitted the RHESSI spectrum shown in Fig. 14 with a collisional thick target model of a powerlaw of accelerated electrons with spectral index $\delta$ (i.e. $f(E) \propto E^{-\delta}$ ) above a low energy cutoff $E_{\mathrm{C}}[\mathrm{keV}]$ of total number flux $N_{\mathrm{e}}=$ $\int f(E) \mathrm{d} E$ [electrons s $^{-1}$ ] (Brown 1971; Holman 2003). From this the total power in the electron beam can be calculated as

$P=\int f(E) E \mathrm{~d} E=1.6 \times 10^{-9} N_{\mathrm{e}} E_{\mathrm{C}} \frac{\delta-1}{\delta-2}$

with the power in units of $\operatorname{erg~s}^{-1}$. Using the fitted parameters shown in Table 2 we find that the power in non-thermal electrons at 16:02:40 is $(7.7 \pm 2.9) \times 10^{27} \mathrm{erg} \mathrm{s}^{-1}$ and at 16:09:52 (7.8 \pm
2.2) $\times 10^{27} \mathrm{erg} \mathrm{s}^{-1}$. Since both times return very similar values we shall use the earlier time in the following as it corresponds better with the peak velocity flows.

Power per unit area, $F_{\mathrm{P}}$ is obtained from an estimate of the HXR source size. Both panels of Fig. 13 show that the 12-25 keV sources are compact and comparable in width to the EUV ribbon enhancements, which are around 5" wide. We take the EUV ribbon width as an estimate for the HXR source diameter. Sources at $18-25 \mathrm{keV}$ are more point-like than the lower energy sources, so we further propose that the majority of the non-thermal beam power is delivered in an area of $5^{\prime \prime} \times 5^{\prime \prime}$ or $1.3 \times 10^{17} \mathrm{~cm}^{2}$. The power per unit area, $F_{\mathrm{P}}$ is thus around $(5.9 \pm 2.2) \times 10^{10} \mathrm{erg} \mathrm{cm}^{-2} \mathrm{~s}^{-1}$ above $15.6 \mathrm{keV}$. With this value of $E_{\mathrm{C}}$, beam energy is mostly deposited around $5-10 \times 10^{19} \mathrm{~cm}^{-2}$. Note, because the spectral index is so steep, a small increase in the low-energy cutoff value would change the power by a large factor (the power above $E_{\mathrm{C}}$ depends on $E_{\mathrm{C}}^{2-\delta}$ ).

We arrive at a number for the beam power based on spectral fitting comparable with the values of $F_{\mathrm{P}} \sim 10^{10} \mathrm{erg} \mathrm{cm}^{-2} \mathrm{~s}^{-1}$ at which explosive evaporation occurs in modelling, although there is probably sufficient leeway to be consistent also with the values needed for a gentle flow. Furthermore, as mentioned above there are strong flow locations showing no HXR sources which will therefore have $F_{\mathrm{P}}$ values reduced by a factor corresponding roughly to RHESSI's dynamic range (a factor 10) or more.

Electron beam heating. In the VAL-E model atmosphere, which we assume to be a reasonable pre-flare atmosphere, the temperature at a density of a few $\times 10^{10} \mathrm{~cm}^{-3}$ is around $20000 \mathrm{~K}$. To produce the $\mathrm{Fe}$ line emission observed requires heating to $1.5 \times 10^{6}$ K. From Eq. (36) in Emslie (1978) we have the following expression for the thick-target beam collisional heating rate per target particle, at column depth $N$ due to beam electrons above $E_{\mathrm{c}}$ in a fully ionised hydrogen plasma.

$Q=\pi \Lambda e^{4}(\delta-2) B\left(\frac{\delta}{2}, \frac{1}{3}\right) \frac{F_{\mathrm{P}}}{E_{\mathrm{C}}^{2}}\left[\frac{6 \pi \Lambda e^{4} N}{E_{\mathrm{C}}^{2}}\right]^{-\delta / 2}$

where $B$ is the Beta function. Inserting measured values of $\gamma$ and $E_{\mathrm{C}}$, and using $N=5.8 \times 10^{18} \mathrm{~cm}^{-2}$ (i.e. where $n_{\mathrm{e}} \sim$ $4 \times 10^{10} \mathrm{~cm}^{-3}$ in the VAL-E atmosphere) we see that the heating rate per particle is $8.1 \times 10^{-16} F_{\mathrm{P}} \mathrm{erg} \mathrm{s}^{-1} \sim 3.4 \times 10^{-4} \mathrm{erg} \mathrm{s}^{-1}$. This is well in excess of the radiative energy loss rate per particle, $n_{\mathrm{e}} f(T)$ (where $f(T)$ is the radiative loss function, $f(T)<$ $10^{-21} \mathrm{erg} \mathrm{s}^{-1} \mathrm{~cm}^{3}$, Martens et al. 2000) or the conductive energy loss rate per particle $\sim 10^{-6} T^{7 / 2} / n_{\mathrm{e}} h^{2}$ for $T \sim 2 \times 10^{6} \mathrm{~K}$ and $h \sim 10^{7} \mathrm{~cm}$, approximately the transition region scale height. So the plasma will heat quickly.

The double thermal fit parameters suggests we see around $5 \%$ of the plasma at a temperature of $26 \mathrm{MK}$. From a simple calculation using the same footpoint size estimate, and the emission measure of the hot component, the depth of the emitting region should be around $1500 \mathrm{~km}$, on the order of the depth of the chromosphere. This does help confirm that there are hot footpoints present, but unlike the non-thermal fit, gives us no real insight into the energy deposition method.

Flow speeds. We can compare the spectroscopicallydetermined line-of-sight flow speeds observed in Figs. 10 to 12 to the relevant sound speed $c_{\mathrm{s}}$. The sound speed is

$C_{\mathrm{s}}=9.79 \times 10^{5}\left(\frac{\gamma Z T_{\mathrm{e}}}{\mu}\right)^{\frac{1}{2}}$ 
where for ionised hydrogen $Z=1, \gamma=\frac{5}{3}$ and $\mu=1$. Taking the temperature of formation of the Fe XIII line as $T_{\mathrm{e}}=1.58 \mathrm{MK}$, the sound speed of the plasma is $c_{\mathrm{s}}=147 \mathrm{~km} \mathrm{~s}^{-1}$, and for Fe XVI $c_{\mathrm{s}}=185 \mathrm{~km} \mathrm{~s}^{-1}$. Our observational upper limit of $140 \mathrm{~km} \mathrm{~s}^{-1}$ is smaller than, though reasonably consistent with these speeds. What is perhaps best demonstrated by these findings is that in a flare of relatively small energy such as this, there is no clear cut case for either evaporation scenario, when the behaviour of individual footpoints across the region is so diverse.

\section{Conclusions}

In this paper we have presented a detailed application of Hinode/EIS density diagnostics to the footpoint regions of a solar flare. To aid in interpretation, we selected a flare with cotemporal TRACE and RHESSI observations. We find evidence for an increase in the density of flare footpoint plasma plasma emitting at Fe XII-Fe XIV temperatures of $\sim 1.5-2 \mathrm{MK}$, consistent with a downwards motion of the transition region during gentle evaporation. RHESSI spectroscopy interpreted in the collisional thick target model allows us to put some limits on the energetics of an electron beam producing the flare-related heating, though the high background and very soft spectrum in this event, coupled with the usual uncertainty in the low energy cutoff, make these somewhat speculative. Using fit parameters obtained and an estimate of the RHESSI non-thermal source size produces a beam power per unit area in excess of that which should lead to explosive evaporation. Alternatively, the spectra have been interpreted using a double thermal model, which fit the data equally well, and place the heating within a region comparable to depth of the chromosphere. Imaging at two periods in the impulsive phase also suggests the HXR emission is dominated by footpoint sources and not loop structures heated to flare temperatures, although the spectral fits do not allow us to determine if this is either completely thermal or non-thermal emission. The largest signal in our spectral fits originates from slow-moving plasma, with speeds substantially less than the predicted explosive evaporation speeds, but we do find evidence for a weaker rapid upflow component, consistent with explosive evaporation. The largest speeds do not occur co-spatially with the RHESSI sources. However, the total beam energy depends sensitively on the electron low energy cutoff which is extremely difficult to determine, especially in a weak event like this. The electrons producing the observed HXR emission are easily capable of penetrating to the partially neutral atmosphere, where they will increase the ionisation fraction as well as heating the plasma, which may also be in part responsible for the observed electron density changes.

Time-resolved studies such as these provide observational constraints on models of chromospheric beam heating and evaporation. In the event presented, the beam parameters are too ill-defined to be very useful, but future observations, particularly with an increased range of density diagnostics, should help pin down the evolution of the transition region and upper chromosphere during a flare, and test the available models.

Acknowledgements. We are grateful to the anonymous referee whose critical comments led to improvements in this work, and to Ryan Milligan and Peter Young who gave valuable input to our analysis. DRG acknowledges support from an STFC-funded Ph.D. studentship. L.F. and I.G.H. acknowledge support from STFC-funded rolling grant ST/F002637/1. This work was also supported by the EC's SOLAIRE Research and Training Network at the University of Glasgow (MTRN-CT-2006-035484) and by Leverhulme Foundation Research Grant F/00 179/AY. CHIANTI is a collaborative project involving the NRL (USA), the Universities of Florence (Italy) and Cambridge (UK), and George Mason University (USA). We are grateful for the open data policies of RHESSI, TRACE and Hinode and the efforts of the instrument and software teams. Hinode is a Japanese mission developed and launched by ISAS/JAXA, with NAOJ as domestic partner and NASA and STFC (UK) as international partners. It is operated by these agencies in co-operation with ESA and NSC (Norway). We also thank NASA for its support of the RHESSI and TRACE programmes.

\section{References}

Allred, J. C., Hawley, S. L., Abbett, W. P., \& Carlsson, M. 2005, ApJ, 630, 573 An, C., Canfield, R. C., Fisher, G. H., \& McClymont, A. N. 1983, ApJ, 267, 421 Antiochos, S. K., \& Sturrock, P. A. 1978, ApJ, 220, 1137

Brown, J. C. 1971, Sol. Phys., 18, 489

Brown, J. C. 1973a, Sol. Phys., 29, 421

Brown, J. C. 1973b, Sol. Phys., 31, 143

Brown, C. M., Feldman, U., Seely, J. F., Korendyke, C. M., \& Hara, H. 2008, ApJS, 176, 511

Culhane, J. L., Harra, L. K., James, A. M., et al. 2007, Sol. Phys., 243, 19

Czaykowska, A., Alexander, D., \& De Pontieu, B. 2001, ApJ, 552, 849

De Pontieu, B., McIntosh, S. W., Hansteen, V. H., \& Schrijver, C. J. 2009, ApJ, 701, L1

Del Zanna, G., Mitra-Kraev, U., Bradshaw, S. J., Mason, H. E., \& Asai, A. 2011 A\&A, 526, A1

Dennis, B. R., \& Zarro, D. M. 1993, Sol. Phys., 146, 177

Dere, K. P., Landi, E., Young, P. R., et al. 2009, A\&A, 498, 915

Emslie, A. G. 1978, ApJ, 224, 241

Fisher, G. H., Canfield, R. C., \& McClymont, A. N. 1985a, ApJ, 289, 425

Fisher, G. H., Canfield, R. C., \& McClymont, A. N. 1985b, ApJ, 289, 414

Golub, L., Deluca, E., Austin, G., et al. 2007, Sol. Phys., 243, 63

Handy, B. N., Acton, L. W., Kankelborg, C. C., et al. 1999, Sol. Phys., 187, 229

Hannah, I. G., Hudson, H. S., Hurford, G. J., \& Lin, R. P. 2010, ApJ, 724, 487

Harra, L. K., Sakao, T., Mandrini, C. H., et al. 2008, ApJ, 676, L147

Harrison, R. A., Sawyer, E. C., Carter, M. K., et al. 1995, Sol. Phys., 162, 233

Holman, G. D. 2003, ApJ, 586, 606

Hudson, H. S. 1972, Sol. Phys., 24, 414

Kane, S. R., \& Anderson, K. A. 1970, ApJ, 162, 1003

Lin, R. P., Dennis, B. R., Hurford, G. J., et al. 2002, Sol. Phys., 210, 3

Martens, P. C. H., Kankelborg, C. C., \& Berger, T. E. 2000, ApJ, 537, 471

Milligan, R. O. 2008, ApJ, 680, L157

Milligan, R. O., \& Dennis, B. R. 2009, ApJ, 699, 968

Milligan, R. O., Gallagher, P. T., Mathioudakis, M., et al. 2006a, ApJ, 638, L117

Milligan, R. O., Gallagher, P. T., Mathioudakis, M., \& Keenan, F. P. 2006b, ApJ, 642, L169

Mrozek, T., \& Tomczak, M. 2004, A\&A, 415, 377

Neupert, W. M. 1968, ApJ, 153, L59

Ricchiazzi, P. J., \& Canfield, R. C. 1983, ApJ, 272, 739

Tsuneta, S., Ichimoto, K., Katsukawa, Y., et al. 2008, Sol. Phys., 249, 167

Vernazza, J. E., Avrett, E. H., \& Loeser, R. 1981, ApJS, 45, 635

Warren, H. P., Feldman, U., \& Brown, C. M. 2008, ApJ, 685, 1277

Watanabe, T., Hara, H., Sterling, A. C., \& Harra, L. K. 2010, ApJ, 719, 213

Young, P. R., Del Zanna, G., Mason, H. E., et al. 2007, PASJ, 59, 857

Young, P. R., Watanabe, T., Hara, H., \& Mariska, J. T. 2009, A\&A, 495, 587 